\title{
Vendaje neuromuscular: Efectos neurofisiológicos y el papel de las fascias
}

\author{
Neuromuscular Bandage: Neurophysiological Effects and the Role of Fascias \\ Bandagem neuromuscular: Efeitos neurofisiológicos e o papel das fáscia
}

Ximena María Villota Chicaíza'

Doi: dx.doi.org/10.12804/revsalud12.2.2014.08

Para citar este artículo: Villota-Chicáiza XM. Vendaje neuromuscular: Efectos neurofisiológicos y el papel de las fascias. Rev Cienc Salud. 2014;12(2): 253-69.

doi: dx.doi.org/10.12804/revsalud12.2.2014.08

\begin{abstract}
Resumen
Durante los últimos años, el vendaje neuromuscular, un aplicativo terapéutico creado en 1979 por el doctor Kenzo Kase, ha venido introduciéndose en el manejo de muchas alteraciones del sistema musculo-esquelético y más aún en el tratamiento de trastornos neurológicos; esta herramienta terapéutica que consiste en un vendaje elástico autoadhesivo permite la recuperación de la parte lesionada sin disminuir su función corporal. De acuerdo con la literatura existente sobre los efectos fisiológicos producidos por este aplicativo terapéutico en el organismo, se podría decir que existe consenso. Sin embargo, en este artículo la autora quiere destacar el significativo aunque poco resaltado papel que juegan las fascias en los efectos terapéuticos del vendaje neuromuscular, analizando desde una perspectiva reflexiva el efecto analgésico, neuromecánico y circulatorio, como efectos fundamentales del vendaje neuromuscular y la función de las fascias en los mismos, con lo que intenta aportar un entendimiento global en la manera como se relacionan todos los tejidos conectivos, aspectos que son de gran importancia tanto para la evaluación de alteraciones como para la prescripción adecuada del vendaje neuromuscular.
\end{abstract}

Palabras claves: vendaje neuromuscular, neurofisiológico, efecto analgésico, efecto neuromecánico, efecto circulatorio, fascias, receptor, propiocepción.

\section{Abstract}

During the last years, neuromuscular bandage, a therapeutic application created in 1979 by doctor Kenzo Kase has been introduced in the management of many disorders of the musculo-skeletal system and even more so in the treatment of neurological disorders; This therapeutic tool which consists of a self adhesive elastic bandage allows recovery of the injured party without diminish-

1 Universidad Manuela Beltrán. Departamento de Investigaciones. Correspondencia: ximena.villota@virtualumb.com. 
ing its bodily function. According to the existing literature on the physiological effects of this therapeutic application in the body, you could say that there is consensus. However in this article the author wants to highlight the significant although little highlighted role played by the fascias on the therapeutic effects of neuromuscular bandage, analyzing from a reflective perspective the analgesic, neuromechanical and circulatory effects, as fundamental effects of neuromuscular bandage and fascias in the same function, trying to bring a global understanding on the way they relate to all connective tissues, aspects that are of great importance for the proper evaluation of alterations and prescription of neuromuscular bandage.

Keywords: neuromuscular bandage, neurophysiological, analgesic effect, neuromechanical effect, circulatory effect, fascias, receiver, proprioception.

\section{Resumo}

Durante os últimos anos a bandagem neuromuscular, um aplicativo terapêutico criado em 1979 pelo doutor KJenzo Kase tem vindo se introduzindo no maneio de muitas alterações do sistema musculoesquelético e especialmente no tratamento de transtornos neurológicos; esta ferramenta terapêutica que consiste em uma bandagem elástica autoadesiva, permite a recuperação da parte lesada sem diminuir sua função corporal. De acordo com a literatura existente sobre os efeitos fisiológicos produzidos por este aplicativo terapêutico no organismo poderia se dizer que existe consenso. No entanto, neste artigo a autora quer destacar o significativo ainda pouco destacado papel que jogam as fáscias nos efeitos terapêuticos da bandagem neuromuscular, analisando desde uma perspectiva reflexiva o efeito analgésico, neuromecânico e circulatório, como efeitos fundamentais da bandagem neuromuscular e a função das fáscias, tentando aportar um entendimento global na forma como se relacionam todos os tecidos conectivos, aspectos que são de grande importância tanto para a avaliação de alterações quanto para a prescrição adequada da bandagem neuromuscular.

Palavras-chave: bandagem neuromuscular, neurofisiológico, efeito analgésico, efeito neuromecânico, efeito circulatório, fáscias, receptor, propriocepção.

\section{Introducción}

El vendaje neuromuscular mundialmente conocido como kinesiotaping es un revolucionario dispositivo terapéutico que desde los años setenta ha venido introduciendo un nuevo concepto dentro de todos los sistemas de vendajes así como también se ha ido abriendo campos de aplicación desde sus inicios en el rendimiento deportivo hasta una gran cantidad de afecciones musculo-esqueléticas, en el campo de la medicina física y la rehabilitación. Este aplicativo terapéutico puede ser calificado como novedoso, ya que mientras otros tipos de vendaje centran su filosofía en inmovilizar o estabilizar artificialmente las estructuras músculo-articulares y ligamentosas del cuerpo, lo cual puede, en algunos casos, ser contraproducente porque se inhiben los estímulos, debilitan la musculatura y disminuyen el riego sanguíneo, aspectos que influyen negativamente en el proceso de rehabilitación del sistema osteomuscular; el vendaje neuromuscular fue diseñado para facilitar el proceso de curación natural del cuerpo permitiendo apoyo y estabilidad a los músculos 
y las articulaciones sin restringir el alcance del movimiento del cuerpo, el vendaje neuromuscular es en sí mismo un estímulo para incidir a todos los sistemas del organismo y facilitar los procesos de curación (1).

Este vendaje tiene unas características muy similares a las de la piel en cuanto a grosor y elasticidad; "se trata de un esparadrapo elástico constituido en un $100 \%$ por una estructura trenzada de hilos de algodón, que incorpora una capa de pegamento llamado cyanoacrilato de uso médico que le confiere adhesividad. Esta capa de pegamento es antialérgica, no contiene látex e imita la huella dactilar para favorecer la transpiración y la elevación de la piel. El vendaje se encuentra adherido a un papel protector, dotado desde fábrica de un característico preestiramiento conseguido por la forma especial de unir el vendaje con el papel protector y que es aproximadamente de un $10 \%$ inicial al que se puede aplicar hasta un $160 \%$ adicional, con lo que se iguala la elasticidad de la piel, en sentido transverso por el contrario es totalmente inelástico" (2).

Para comprender el accionar terapéutico del vendaje neuromuscular es importante tener en cuenta que su creador, el doctor Kenzo Kase, licenciado en quiropráctica y acupuntura, desarrolló su método según los conceptos de medicina asiática, en donde la piel es el órgano reflexógeno más grande del cuerpo y desde el cual se puede incidir con estímulos aferentes hacia el sistema nervioso central desencadenando las respuestas neurofisiológicas deseadas. Los efectos fisiológicos del vendaje neuromuscular son el analgésico, el de aumento de la circulación linfática y sanguínea, reducción de la fatiga muscular y modulación del tono muscular, mejora la interrelación entre las fascias, actúa sobre la postura y la propiocepción articular (1).

Cuando se piensa en el gran auge que ha venido teniendo el vendaje neuromuscular, que fue la sensación en los juegos olímpicos de Beijing de 2008 y de los de Inglaterra en 2012, surge el problema frente a si quienes se han capacitado en el manejo de este aplicativo terapéutico y lo están implementando, conocen los principios neurofisiológicos que median sus efectos terapéuticos, así como la importancia de las fascias como agentes neuromecánicos y los tienen en cuenta a la hora de prescribir el tipo de técnica que le aplicarán a su paciente. Para la autora, el vendaje neuromuscular más que una herramienta terapéutica es un concepto rehabilitatorio, que se fundamenta en la neurofisiología, neuromecánica y fisiología muscular, ciencias a través de las cuales se pueden explicar sus efectos terapéuticos.

Por tanto, en este artículo se presenta desde una perspectiva reflexiva un análisis de los efectos neurofisiológicos del vendaje neuromuscular y del papel que desempeñan las fascias en estos; aspectos que han de tenerse en cuenta no solo para comprender el accionar terapéutico del vendaje neuromuscular sino para realizar una adecuada prescripción del mismo. En una primera parte de este artículo se desarrollan los conceptos anatómicos y neurofisiológicos más importantes sobre la piel y las fascias, para continuar en un segundo apartado analizando el efecto analgésico del vendaje; luego, en un tercer apartado se trata el efecto neuromecánico aplicado a los efectos del vendaje sobre los músculos y articulaciones, continuando con una cuarta parte donde se analiza el efecto del vendaje neuromuscular sobre la circulación sanguínea y linfática. Por último, se brindan las conclusiones sobre el tema.

La piel y las fascias

La piel es el órgano vital más grande de cuerpo, cubre toda nuestra superficie corporal y presenta diferentes grosores en diversas regiones del cuerpo; por ejemplo, en las palmas de las manos y plantas de los pies, es mucho más gruesa, que en regiones como los párpados, 
donde es muy delgada. A su vez, la piel posee diferentes grados de sensibilidad de acuerdo con la distribución topográfica y densidad de los receptores cutáneos; sin embargo, en promedio, un centímetro cuadrado de piel posee cuatro metros de nervios, un metro de vasos y cinco mil órganos sensitivos, incluyendo las terminaciones nerviosas libres que transmiten el dolor y el prurito (3).

Este órgano vital está formado por tres capas, la epidermis, la dermis y la hipodermis o también llamado tejido adiposo subcutáneo. La dermis es mucho más gruesa que la epidermis —aproximadamente de 10 a 30 veces - y en ella se encuentran los vasos arteriales, venosos y linfáticos, los nervios, los músculos erectores del pelo y los folículos pilocebáceos-apocrinos, así como las glándulas ecrinas (4). En la piel hay mecanoceptores denominados corpúsculos de Pacini que se localizan en la dermis profunda o grasa subcutánea y que reaccionan a cambios mecánicos rápidos, los corpúsculo de Meissner, ubicados en la dermis en las regiones palmo-plantares captan la sensibilidad táctil fina; corpúsculos de Ruffini, localizados en la dermis y en las articulaciones, se activan por deformaciones mecánicas; el corpúsculo táctil de Merkel es abundante en regiones de alta capacidad de percepción táctil; las terminaciones nerviosas libres son las más abundantes y son los receptores cutáneos de mayor importancia, estas contribuyen a la sensación táctil, se encuentran en la dermis y en la epidermis; en esta última discurren como axones desnudos entre las células epidérmicas, se cree que detectan los estímulos que generan las sensaciones de dolor y térmicas aunque también muchas son capaces de responder a estímulos mecánicos (4). La estructura de los termoceptores no se ha determinado aunque los hay para frío y calor, se cree que los corpúsculos de Ruffini también participan en la percepción del calor, los bulbos de Krause se localizan en la dermis y aunque su función no se sabe con certeza se cree que responden a estimulación mecánica de la piel como también a estímulos de frío (figura 1).

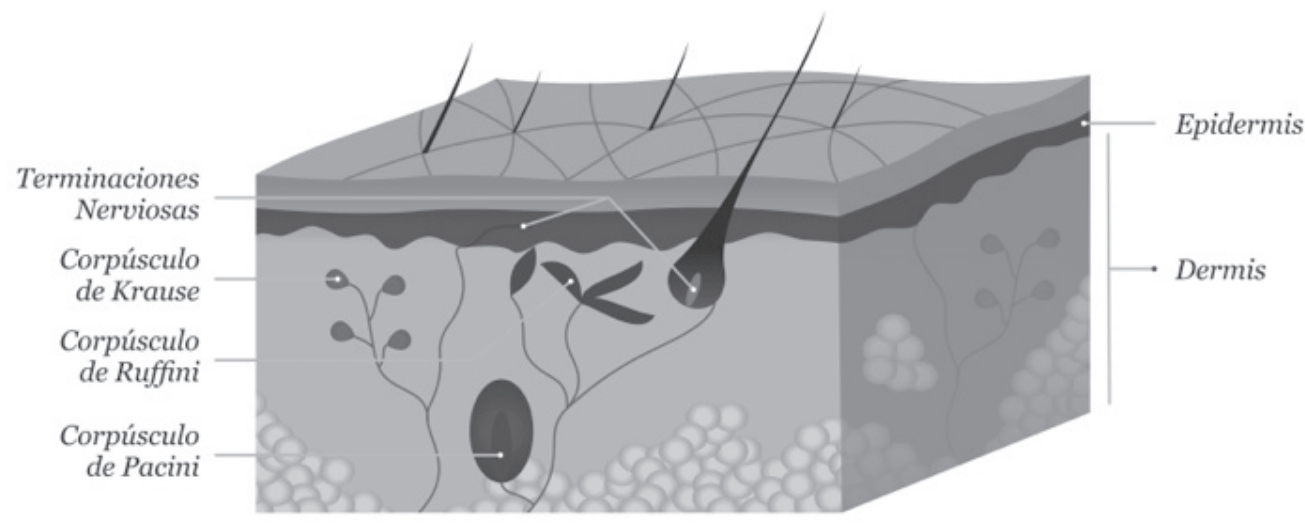

Figura 1. Distribución de receptores en la piel

Ilustración: Universidad Manuela Beltrán. 
Algo que sí parece estar claro en el sistema sensorial cutáneo es que algunos receptores pueden captar varios tipos de sensaciones, esta alta sensibilidad cutánea permite comprender la gran aferencia sensorial con la que se puede influir al sistema nervioso central a través de la estimulación de la piel.

De otra parte, para poder comprender los efectos del vendaje neuromuscular es muy importante resaltar le estrecha interrelación que existe entre la piel y las fascias (figura 2) y de estas últimas con todos los tejidos corporales principalmente los del aparato musculo-esquelético. Las fascias son una serie ininterrumpida de tejidos conectivos resistentes pero retráctiles que se encuentran desde la cabeza a los pies y desde el exterior al interior del cuerpo, un tejido continuo que envuelve todo el organismo y todas las estructuras en él contenidas; teniendo en cuenta que la porción más profunda de la piel, la hipodermis, se une a la fascia superficial, los estímulos y efectos mecánicos generados en la piel se trasmiten a las fascias superficiales y de estas a las profundas. Los estudios anatómicos e histológicos han comprobado que las fascias se encuentran en todas las regiones del cuerpo y que, a su vez, están formadas por diferentes capas, de dirección oblicua, transversal o circular por lo que el aspecto general de las fascias es de espiral (5).

Estas tienen un origen embrionario mesodérmico por lo que guardan una estrecha relación con casi todos los tejidos del cuerpo que tienen el mismo origen (6). El sistema fascial superficial está formado por una red que se extiende desde el plano subdérmico hasta la fascia muscular. Se compone de numerosas membranas horizontales, muy finas, separadas por cantidades variables de grasa y conectadas entre sí a través de los septos fibrosos del recorrido vertical u oblicuo. En su recorrido profundo, la fascia superficial, de modo similar, se conecta con el sistema miofascial, formando junto con este una unidad funcional (7). Por tanto, los estímulos y acciones mecánicas producidas por el vendaje neuromuscular sobre la piel, son transmitidos a las fascias y de estas a los músculos.

Contrario a lo que se creía anteriormente, el tejido fascial es un sistema activo ya que la fascia es un tejido altamente sensible, investigaciones sobre su microestructura apoyan que existe una abundante red nerviosa de recepto-

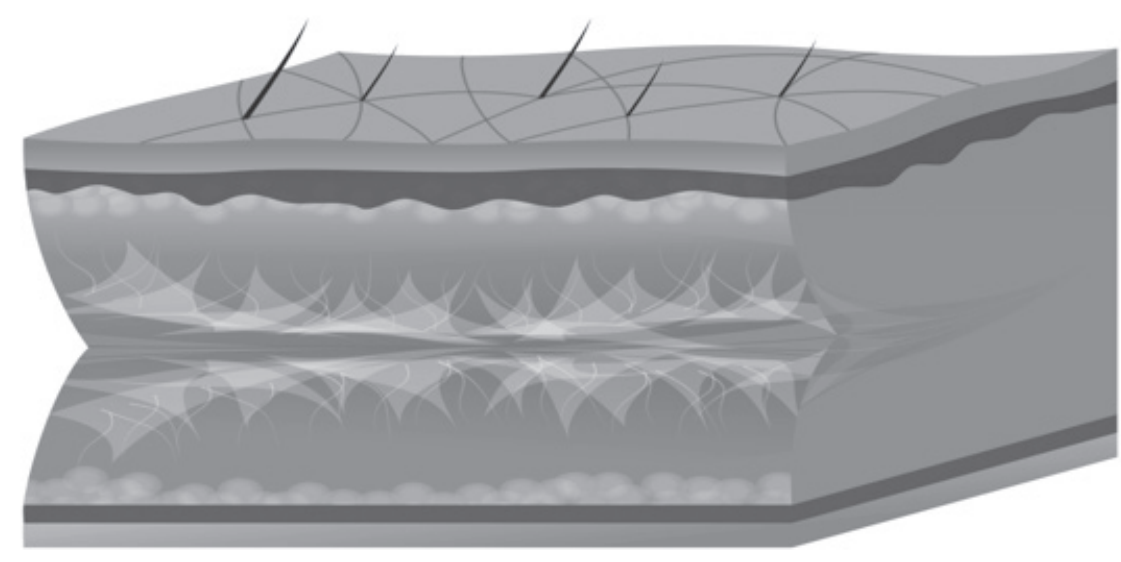

Figura 2. La fascias

Ulustración: Universidad Manuela Beltrán. 
res intrafasciales (de Golghi) y células musculares lisas propias del tejido fascial, lo que puede sugerir que la fascia tiene capacidad de tener actividad propia (8). Por lo que sería lógico pensar que pueden captar estímulos externos $\mathrm{y}$ tener sus propios movimientos y reacciones.

Las funciones de las fascias son múltiples e importantes. Entre las principales funciones de la fascia superficial, a parte de su función nutritiva, se destacan el soporte y la definición de los depósitos de la grasa del tronco y de las extremidades, así como también el sostén de la piel con referencia a los tejidos subyacentes. Es un aspecto importante, ya que la suspensión del sistema fascial superficial controla el contorno corporal estático y dinámico, por tanto, los cambios favorables y desfavorables en el comportamiento funcional estático y dinámico del sistema fascial superficial influyen directamente en la mecánica del sistema miofascial musculo-esquelético donde cada una de sus partes se encuentra influida por la otra (7). Este aspecto resulta relevante para explicar cómo las tensiones ejercidas por el vendaje neuromuscular sobre la fascia superficial, a través de la piel, pueden finalmente influir en la mecánica del músculo.

La fascia profunda está constituida por un material más fuerte y denso que el que constituye la fascia superficial. Su grosor y densidad dependen de la ubicación y la función específica que desempeña. A medida que aumenta la exigencia de las necesidades mecánicas se densifica la estructura del colágeno, su principal componente. Basándose en la densidad del tejido colágeno, la fascia se puede dividir según su función, en el tejido de unión, de revestimiento, de sostén y de transmisión (7).

El sistema fascial profundo soporta, rodea y asegura la estructura e integridad de los sistemas muscular, visceral, articular, óseo, nervioso y vascular. La fascia profunda cubre las áreas corporales como si fueran enormes envolturas, protegiéndolas y dándoles forma (7). Como ya se había dicho anteriormente la fascia superficial y la profunda se encuentran íntimamente unidas a través de conexiones fibrosas, por ende las tensiones mecánicas que afectan a una, también afectan a la otra.

Sin embargo, el interés particular aquí es resaltar la estrecha conexión de las fascias con los músculos a los cuales envuelve a través de los perimisios; de su asociación con los vasos sanguíneos y linfáticos los cuales perforan las fascias para poder entregar el aporte vásculonervioso a los diferentes órganos y tejidos, y de la indisolubilidad de las fascias con los ligamentos, cartílagos y huesos que se adaptan íntimamente con ellas y que, a su vez, son prolongaciones o densificaciones de las mismas; porque esto permite entender cómo actúa el vendaje neuromuscular sobre todos los tejidos bajo la piel, ya que los efectos mecánicos generados por el vendaje sobre la misma, se trasmiten hacia los demás tejidos que se interconectan con ella, como las fascias, tanto superficiales como profundas que se encargan de trasmitir estos efectos a los tejidos musculares, ligamentosos y articulares. La fascia, entonces, es el elemento que nos proporciona la noción de 'globalidad' del aparato locomotor, al conectar los músculos entre sí formando grupos funcionales, y que también une esos grupos funcionales con otros anatómicamente muy separados entre sí; por ello, cuando se realiza un movimiento en un segmento determinado de nuestro cuerpo, este responde como un todo; se realizan reacciones en cadena, inclusive en los lugares más remotos cruzando la línea media del cuerpo y estableciendo las conexiones menos esperadas, siendo la fascia el único tejido que tiene la capacidad de realizarlas (8).

Esta estrecha integridad entre la piel y las fascias que conectan los músculos, los liga- 
mentos, los tendones y articulaciones, sugiere el papel comunicador que tienen las fascias de los estímulos generados por el vendaje neuromuscular, sobre todos los elementos del aparato esqueleto-motor.

De igual manera, este aspecto de que todos los tejidos corporales se encuentran relacionados entre sí por medio de las fascias, permite comprender por qué cualquier alteración funcional o mecánica, en cualquier región del cuerpo, termina repercutiendo en el funcionamiento de órganos y tejidos circunvecinos, así como también las alteraciones en la motilidad y la función de las fascias pueden producir desórdenes orgánicos y biomecánicos, aspectos que se deben tener en cuenta al momento de diagnosticar las alteraciones musculo-esqueléticas, ya que las alteraciones funcionales de una determinada región pueden tener origen en disfunciones fasciales a nivel distal. Hoy día, con el avance en el estudio de las fascias, podemos darle un entendimiento más completo al funcionamiento del organismo y reconocer en sus alteraciones la causa de muchas afecciones orgánicas o músculo-esqueléticas. Sin duda quedan muchas cosas por saber sobre el papel que juegan las fascias en nuestro organismo pero por ahora están definidas sus funciones de soporte, protección, defensa, amortiguación, comunicación, hemodinámica y bioquímica.

\section{Efectos del vendaje neuromuscular}

\section{Efecto analgésico}

Cuando el aparato osteomuscular es sometido a estiramientos, contracciones musculares por sobresolicitación, sobrecargas articulares y descompensaciones a lo largo del día, aparecen las contracturas, los espasmos, las restricciones del flujo sanguíneo y linfático, inflamaciones que en definitiva aumentan la presión intersticial, este aumento activa el estímulo sobre los noci- ceptores los cuales envían aferencias sensoriales dolorosas. Cuando se produce daño al tejido, se liberan o se sintetizan sustancias algogénicas en el mismo tejido lesionado, cuando estas se acumulan en cantidad suficiente, activan los nociceptores o mantienen la excitación; unas activan directamente el nociceptor (bradiquinina, histamina, serotonina), otras no producen dolor por ellas mismas sino que disminuyen el umbral de excitación del nociceptor (prostaglandinas PGE2 y PGI29) y otras alteran la microcirculación local (5). Recordemos que las quininas como la bradiquinina son poderosos vasodilatadores de las arteriolas y que también producen aumento de la permeabilidad capilar lo cual propicia la extravasación de fluidos intracapilares al espacio intersticial lo que produce edema e inflamación (6).

El efecto analgésico del vendaje se da gracias a varios efectos sobre los tejidos afectados; en primer lugar, porque, al aplicar el vendaje, este forma elevaciones sobre la piel llamadas convoluciones lo cual disminuye la presión intersticial y, por ende, la estimulación de los nociceptores; pero este efecto también se debe a la activación del sistema de analgesia natural del organismo a través de las endorfinas y encefalinas que son los analgésicos más potentes que se conocen y sus propiedades se deben a que actúan como neuromoduladores inhibidores, al disminuir la producción de impulsos nerviosos que ascienden por las vías del dolor. El efecto de los nociceptores también se ve disminuido gracias a la normalización de la circulación sanguínea, en primer lugar, y su evacuación linfática posterior, porque los síntomas dolorosos e inflamatorios disminuyen al drenar la acumulación de mediadores inflamatorios de la región afectada como, la bradiquinina, histamina y prostaglandinas, mencionados anteriormente, que sensibilizan los mecanoreceptores y nociceptores haciéndolos hiperexcitables, 
condición en la cual la mínima compresión de los tejidos estimula su input aferente lo que genera sensaciones dolorosas (2).

Otra teoría sobre el efecto analgésico del vendaje neuromuscular, es que la estimulación de los receptores nerviosos de la piel a través del vendaje producen señales de tacto y presión que se trasmiten por las fibras alfa y beta que son rápidas, hasta la sustancia gelatinosa de las astas posteriores de la medula espinal, en donde las fibras $A$ alfa y beta excitan a las células $T$ de la sustancia gelatinosa, con lo que se inhibe la transmisión y cierra la compuerta y se crea un bloqueo en la transmisión del impulso doloroso que viaja por las fibras nociceptoras polimodales $C$ amielínicas, de conducción lenta y que constituye el mecanismo control de compuerta (gate control system). La estimulación de fibras $A$ alfa activa de inmediato los mecanismos centrales. La actividad de estas fibras asciende por los cordones dorsales de la médula espinal y las vías dorso laterales a través del lemnisco medial hacia el complejo ventrobasal del tálamo posterior; de esta manera se proporciona información mucho antes de la llegada de las vías del dolor (9). Por tanto, el efecto analgésico se da porque las fibras alfa y beta que son estimuladas por el vendaje neuromuscular y que son de conducción rápida, llegan primero a los centros nerviosos espinales y supraespinales lo que impide que otras sensaciones, en este caso las dolorosas, sean percibidas.

De otra parte, debemos tener muy en cuenta que las nuevas condiciones fisiológicas que genera el vendaje sobre las regiones afectadas propician los mecanismos curativos naturales del cuerpo, siendo este uno de los principios fundamentales del vendaje neuromuscular. Si miramos hacia los métodos tradicionales de analgesia utilizados en fisioterapia e inclusive en los tratamientos farmacológicos está relacionada a la estimulación de las propiedades autógenas segmentarias y suprasegmentarias de eliminación del dolor o a la inhibición química de los mediadores del proceso inflamatorio y del dolor; pero el vendaje neuromuscular brinda una opción más simple actuando en el origen de las aferencias nociceptivas.

La técnica de 'aumento de espacio' es la indicada para el manejo del dolor, ya que su finalidad es producir una elevación de la piel junto con las fascias superficiales para generar los efectos anteriormente explicados (10). Es muy importante tener claro que cuando un paciente presenta dolor este síntoma debe ser atendido en prelación sobre otros, si el paciente presenta además edema, esta técnica de aumento de espacio se puede combinar con la técnica linfática de la que hablaremos más adelante, ya que, como lo hemos dicho, las condiciones de edema incrementan las aferencias nociceptivas por aumento de la presión intersticial (figura 3).

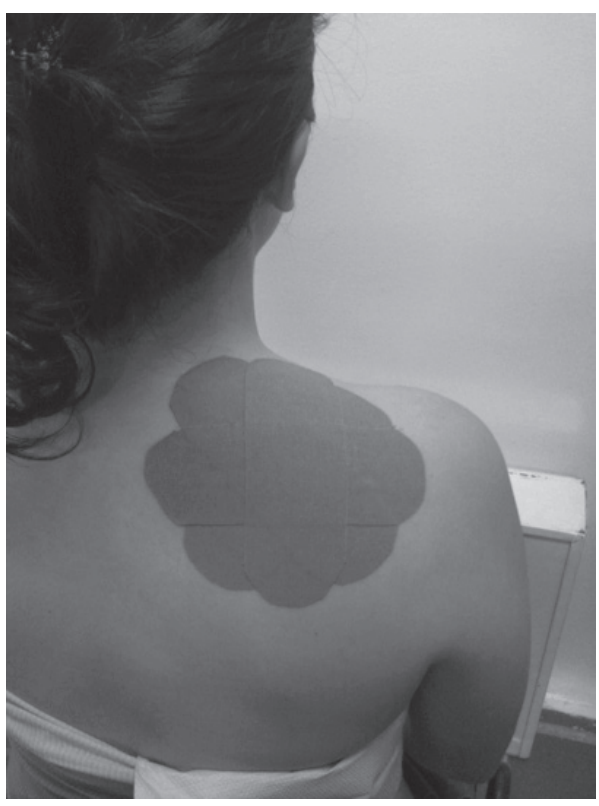

Figura 3. Técnica de aumento de espacio Fotografía: Universidad Manuela Beltrán. 


\section{Efecto neuromecánico}

\section{Músculos}

El sistema muscular no solamente es responsable del movimiento corporal, sino que cumple un papel indispensable en el equilibro global del organismo como promotor circulatorio tanto linfático como sanguíneo y al influir sobre la temperatura corporal (5). Por lo tanto, cuando el funcionamiento muscular se altera, muchos de los sistemas que reciben su influjo directo comienzan a presentar deficiencias. Se han podido encontrar evidencias de múltiples alteraciones orgánicas, óseas, articulares, neurológicas y circulatorias en pacientes con pérdida de la función muscular normal.

El sistema muscular es el motor de las articulaciones, pero, a su vez, es coordinado por la mecánica fascial, el sistema muscular puede funcionar gracias a las fascias, las articulaciones pueden mantener su estabilidad y función a través ellas (5), de hecho la relación entre músculo y articulación se establece a través de tendones y aponeurosis que no son otra cosa que fascias, los ligamentos que estabilizan y protegen las articulaciones son densificaciones de las fascias. Las estrechas interconexiones entre el tejido celular subcutáneo con la epidermis y con las fascias musculares profundas se da a través de las fibras colágenas perpendiculares $\mathrm{y}$ diagonales que intercomunican las diferentes fascias entre sí.

Todo el músculo está rodeado por vainas o fascias de tejido conjuntivo, el epimisio rodea el músculo y se extiende dentro del mismo formando el perimisio que divide el músculo en una serie de fascículos, cada uno de los cuales contiene varias fibras musculares. Dentro del fascículo, las fibras musculares están separadas unas de otras por el endomisio. Todos estos elementos, con proporciones variables de colágeno y fibras reticulares y elásticas, constituyen el componente elástico paralelo a las fibras musculares. El músculo entonces puede ser considerado como una combinación de sus elementos elásticos y contráctiles, el componente elástico, por sus propiedades mecánicas, es similar a los resortes, es decir, para distenderlos hay que aplicar una fuerza (11), y el músculo entonces puede ser distendido; por lo que la tracción que genera el vendaje neuromuscular sobre la piel, al retraerse hacia la base, hace que se produzca un deslizamiento entre las láminas cutáneas, especialmente entre la parte superficial del subcutis y el tejido celular subcutáneo, así se comunica esta tracción al músculo a través de las fascias; este estiramiento dado en las fibras diagonales y perpendiculares del tejido celular subcutáneo activará sus mecanoreceptores los cuales inician un reflejo protector para evitar un estiramiento excesivo entre los tejidos. Este reflejo protector hace que todos los tejidos bajo el subcutis también acompañen el movimiento de la piel. Por tanto, estando la epidermis retraída por el vendaje hacia el origen o inserción muscular, la posición de reposo neurogénico se logra cuando el tejido celular subcutáneo y la fascia muscular también se mueven en dirección a la base del vendaje, y estén en el origen o en la inserción del músculo (figura 4). Este efecto neuromecánico está mediado por la inervación conjunta de la epidermis, la lámina subcutánea, la fascia y el músculo. El estímulo en la parte deslizante profunda (entre el subcutis y la fascia muscular) de deslizamiento en dirección a la base del esparadrapo, provoca un estímulo sobre el músculo de acortamiento o relajación (12). 


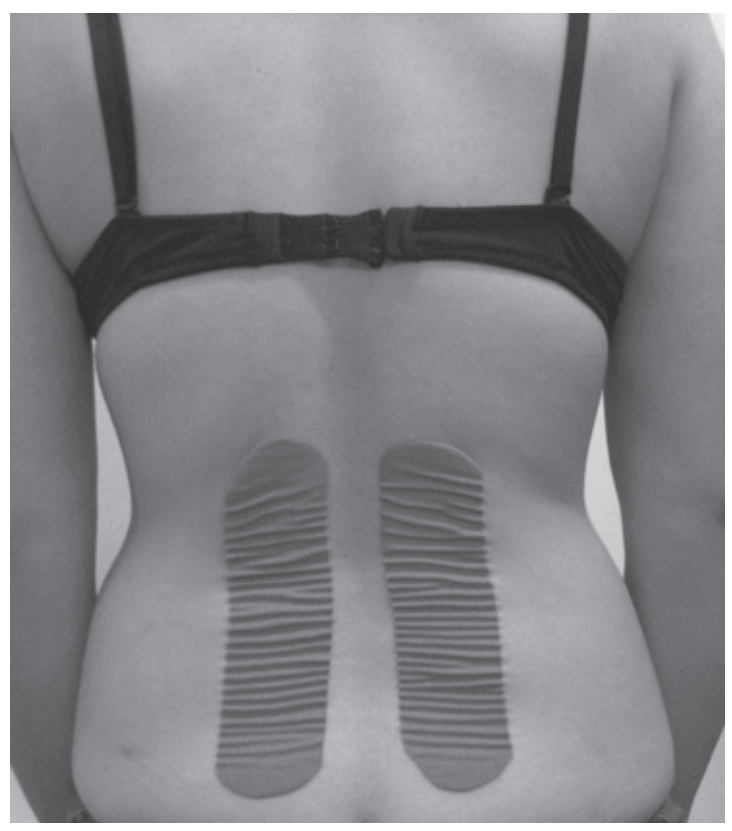

Figura 4. Efecto del vendaje en la técnica muscular

Fotografía: Universidad Manuela Beltrán.

La capacidad de comunicación neuromecánica entre la piel y los músculos permite proveerle al segundo un estado de alargamiento o acortamiento a través del vendaje neuromuscular cuando este se retrae hacia la inserción o el origen del músculo, respectivamente. Este efecto se aplica para inhibir o facilitar un músculo y su utilidad terapéutica es muy amplia, ya que, a través de esto, podemos influir sobre el tono muscular, sobre los espasmos musculares, incrementar o disminuir el input del músculo durante un gesto motor lo cual puede mejorar la fuerza muscular si el estímulo es facilitatorio (el vendaje se retrae hacia el origen) o evitar la lesión de un músculo inhibiendo la sobredemanda del mismo (cuando el vendaje se retrae hacia la inserción del músculo).

Los anteriores efectos podrían ser explicados si analizamos los componentes contráctiles del músculo, estos corresponden a aquellas partes de las sarcómeras donde los filamentos de actina y miosina se deslizan unos sobre otros, en mayor o menor medida, según la intensidad de la contracción. Cuando el músculo se encuentra en estado de reposo, existe solapamiento moderado de los puentes cruzados de miosina sobre actina. En estado de elongación del músculo (cuando el vendaje se retrae hacia la inserción), el solapamiento disminuye y puede llegar a ser nulo, lo cual reduce la capacidad contráctil y de tensión interior del músculo, e influye en la fuerza y tono muscular. Como bien sabemos, durante la contracción el solapamiento o la superposición son máximos y llegan a su límite por el contacto entre los filamentos gruesos y los discos Z (figura 5). La tensión es máxima a la longitud slack o de reposo de la sarcómera (2 um), donde la superposición de la actina sobre la miosina es extrema. La tensión cae progresivamente al aumentar la longitud de la sarcómera, hasta llegar a cero tensión, donde la superposición ya no existe (3,6 um) (11).

\section{Articulaciones}

Como analizamos anteriormente existe una gran variedad de receptores sensoriales que cumplen la función de informar al sistema nervioso central sobre estímulos mecánicos y cambios posturales producidos en el sistema músculo-esquelético. Este mecanismo permite que el sistema nervioso central coordine los movimientos con base en la información que recibe del exterior (somestesia), por un lado, y, por otro, con la información que recibe de los propios órganos (propiocepción). Toda esta información es decodificada y convertida en patrones organizados que posteriormente responderán a la demanda mecánica inducida sobre los segmentos corporales, proporcionando así una respuesta de control y activación muscular (13).

De acuerdo a la distribución de los receptores, tenemos que en las capas superficiales de la cápsula articular, ligamentos cruzados, ligamentos colaterales y cruzados son nume- 


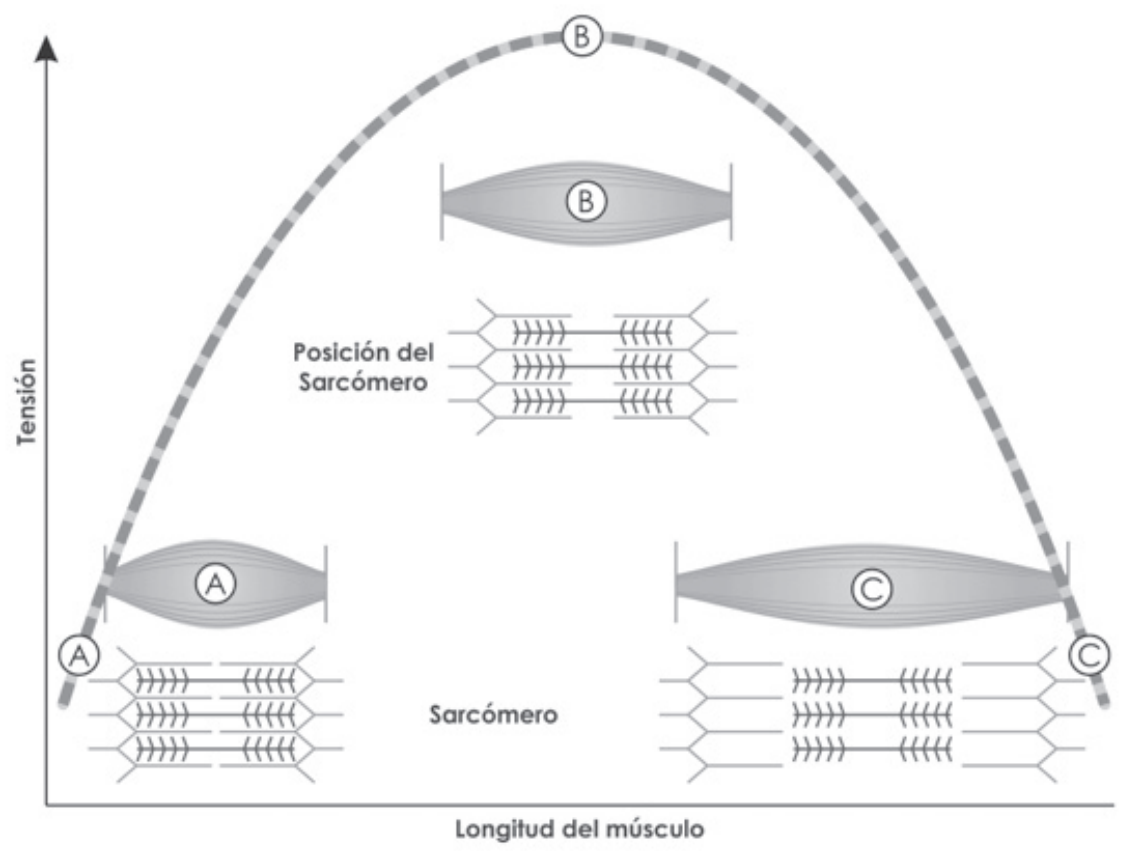

Figura 5. Longitud del músculo y posición de la sarcómera

rosos los corpúsculos de Ruffini (14). En las capas profundas de la cápsula articular, los ligamentos cruzados y colaterales, almohadillas grasas intra y extrarticulares de la rodilla y en el menisco medial se encuentran corpúsculos de Pacini (15). Los discos de Merkel responden a la presión vertical pero no a desplazamientos laterales, los corpúsculos de Meissner son sensibles a los cambios rápidos de presión en áreas pequeñas de la piel, las terminaciones de Ruffini responden a la deformación de la piel y los corpúsculos de Pacini responden rápidamente a la deformación mecánica y la vibración. Todos estos receptores pueden ser estimulados a través de las diferentes técnicas del vendaje neuromuscular, la técnica mecánica aprovecha el componente osteo-ligamentoso por su importante papel transductor de información mecanorreceptiva y propioceptiva que se utiliza para tratar de corregir una posición articular defectuosa o estimular una determinada postura; en esta técnica se aplica con tensión entre $50 \%-75 \%$ realizando una presión hacia adentro y hacia donde queremos reorientar el movimiento articular, tratando de estimular a los propioceptores para desencadenar respuestas en el sistema nervioso central que mejoren la posición y el movimiento articular (figura 6). 


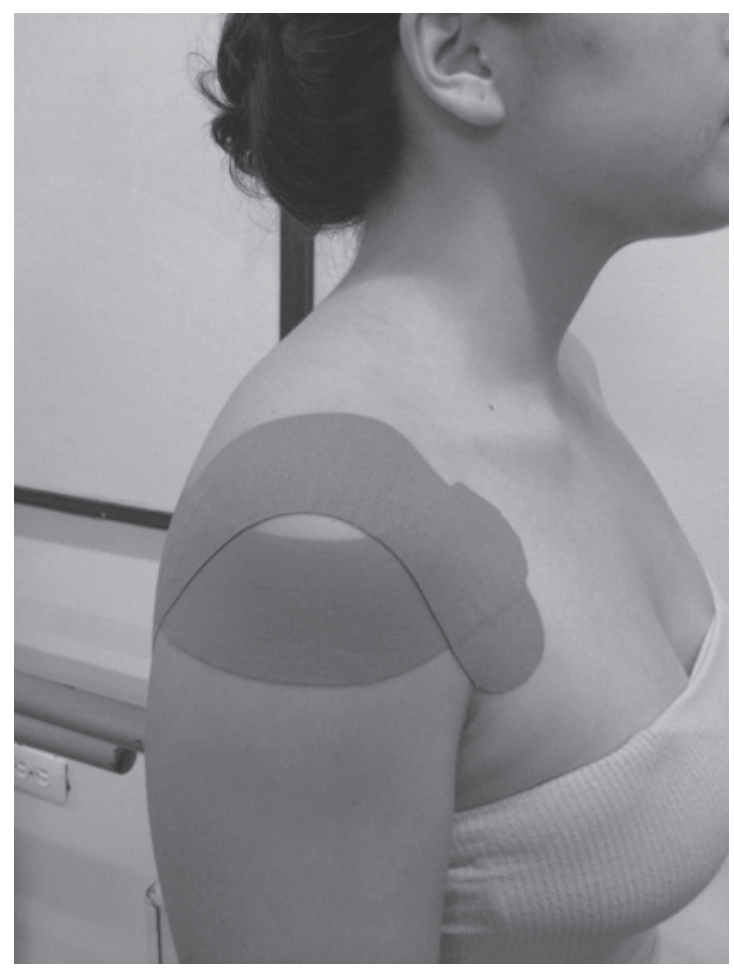

Figura 6. Técnica de corrección mecánica

Fotografía: Universidad Manuela Beltrán.

Las técnicas de ligamento y tendón son técnicas de estímulo propioceptivo, trabajan con tensiones entre $75 \%-100 \%$ y $50 \%-75 \%$, respectivamente; procuran soporte a los ligamentos y tendones lesionados (16) brindan una percepción de apoyo y estabilidad, estimulando los mecanoreceptores y todos los receptores de piel, fascias y músculos que se juntan suministrando abundante información al sistema nervioso que influye en la regulación del movimiento normal. Como sabemos, el movimiento corporal humano está regulado por diferentes estímulos sensoriales entre ellos los propioceptivos que, a su vez, retroalimentan y permiten realizar adecuaciones posturales o motrices; el vendaje neuromuscular actúa como un estímulo sensorial que incentiva posturas y movimientos más fisiológicos y efectivos; a todas las señales aferentes y eferentes y los componentes de integración central y de procesamiento envueltos en el mantenimiento de la estabilidad articular funcional se le denomina sistema sensoriomotor (17) (figura 7).

La técnica de ligamento hace especial énfasis en que los ligamentos deben ser considerados no solo como estructuras mecánicas, sino también como activos comunicadores de la información sensorial (18). En casos de lesiones, la aferencia de las estructuras fasciales y ligamentosas a través del estímulo del vendaje neuromuscular puede ayudar notablemente a la disminución de los síntomas y a la recuperación de la lesión, ya que el vendaje provee soporte a los tejidos lesionados lo que permite que los procesos curativos del cuerpo operen de manera más efectiva.

El vendaje proporciona, de acuerdo con la técnica que se aplica, un estímulo específico para influir sobre la piel, las fascias, los músculos y, a través de estos, sobre las articulaciones porque todos los tejidos están íntimamente relacionados a través de las fascias; por tanto, el vendaje neuromuscular se utiliza para mejorar la alineación conjunta afectando los músculos y la fascia, lo que reduce el mal funcionamiento de las articulaciones e influye en la biomecánica muscular y la movilidad articular. Tengamos siempre presente que las aponeurosis y cápsulas articulares son fascias y están íntimamente conectadas con las superficies articulares, ligamentos, músculos y tendones, "gracias a las fascias las articulaciones pueden mantener su estabilidad y función" (5). 


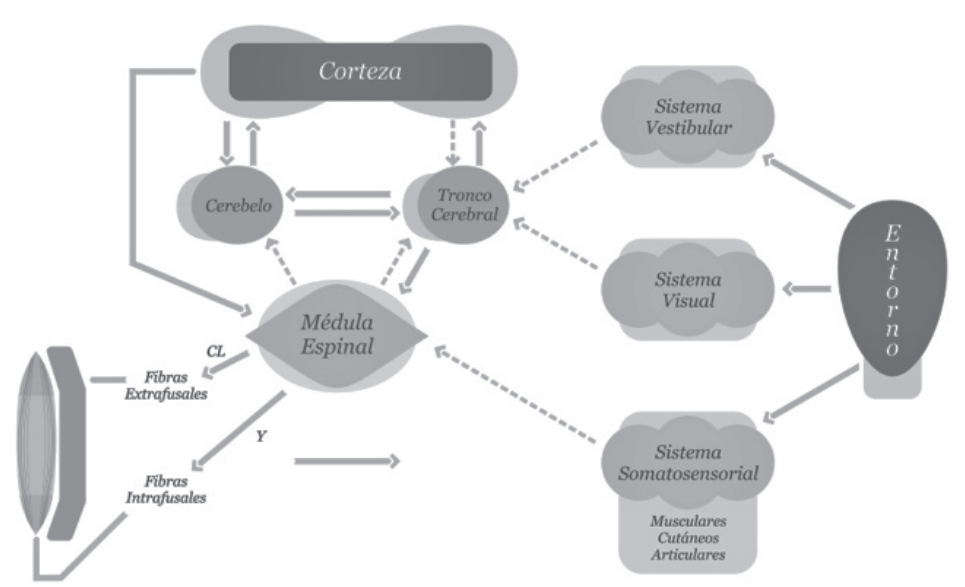

Figura 7. Sistema sensoriomotor

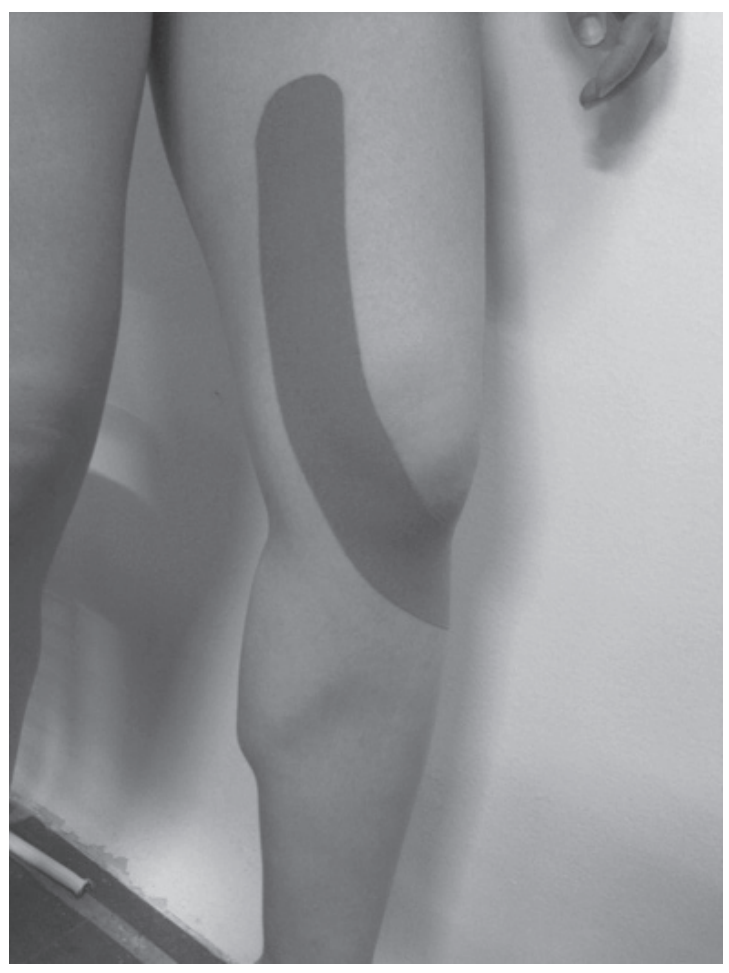

Figura 8. Técnica de ligamento
Efecto circulatorio

Cuando los tejidos han sufrido un trauma se desencadena un proceso de tipo inflamatorio, en el desarrollo de este proceso se producen cuatro eventos fisiológicos fundamentales: vasodilatación, incremento de la permeabilidad microvascular, activación y adhesión celulares, y coagulación. Las citocinas son los mensajeros fisiológicos de la respuesta inflamatoria junto con el proceso inflamatorio y el incremento de la permeabilidad microvascular se forma edema local lo cual presiona sobre los tejidos adyacentes (19). Este aumento de presión trastorna la circulación sanguínea e impide la evacuación linfática, de modo que aumenta la presión en los nociceptores. El cuerpo entiende esto como dolor (20).

Pero la inflamación y el edema no solo se pueden dar a consecuencia de un trauma, también pueden ocurrir por una infección o reacción autoinmune, el problema central en el caso del edema linfático se encuentra en la lámina subcutánea del tejido, generalmente hay un incremento en la circulación sanguínea que el sistema 
linfático es incapaz de subsanar, el resultado será la acumulación de líquidos en el espacio extravascular y el aumento de la presión intersticial, este fenómeno inhibe la función de los vasos linfáticos y sanguíneos lo que incrementa el edema. Algo muy importante de resaltar aquí es que la fascia y los vasos linfáticos tienen una íntima relación entre sí, las fascias son el soporte del sistema nervioso, vascular y linfático, el sistema nervioso y el vascular son interdependientes del sistema fascial, posibilitan la circulación de retorno venoso y linfático, y poseen movimientos ininterrumpidos cuya frecuencia es de unos 8 a 12 periodos por minuto, dichas contracciones actúan como una bomba impelente que permite la circulación de los líquidos por lo que las restricciones o movimientos del tejido fascial pueden modificar el flujo circulatorio (5).

Cuando hay aumento de la presión intersticial los movimientos de la piel y las fascias se disminuyen, las fibras de elastina, reticulina y de colágeno también llamadas biopolímeros contenidas en la matriz fascial son capaces de retraerse debido a una presión superior a la fisiológica para la que su composición biomolecular ha sido concebida y de recuperar su longitud inicial si la presión del medio intersticial vuelve a ser fisiológica (5). Por tanto, lo que se persigue con el vendaje neuromuscular es aumentar el espacio intersticial a través de la elevación de la piel, lo cual no solo permite un mejor tránsito sanguíneo y linfático sino que habilita al tejido fascial para que recupere su motilidad y función, la acción de movilizar la epidermis sobre la dermis genera un espacio que disminuye inmediatamente la presión, y se restablece la circulación sanguínea y la función de evacuación de exceso de líquidos y macromoléculas por parte del sistema linfático (21).
En la técnica linfática del vendaje neuromuscular se coloca la base sin tensión para luego estirar la piel de la zona a tratar mediante una postura adecuada y a continuación se aplica el vendaje. Al volver a la posición de reposo la elasticidad del vendaje hace que se levante ligeramente la piel esto hace que disminuya la presión sobre la dermis, al tiempo que la epidermis es retraída en dirección a la base del vendaje, influenciando sobre las bandas de fijación que permiten la apertura de las uniones intercelulares de los capilares linfáticos iniciales y la entrada de macromoléculas, células y agua (20). Teniendo en cuenta que la apertura y cierre de las uniones que forman el capilar inicial se ven influenciados por la presión tisular total y los cambios de $\mathrm{pH}$ producidos en el intersticio (22).

En esta técnica, la tira del vendaje se fracciona en seis u ocho colas, dependiendo del tamaño y la zona a tratar, tomando aproximadamente tres centímetros para la base, la cual se coloca sin tensión en la cadena ganglionar más cercana, y las tiras se van situando una a una en forma de espiral, de proximal a distal con una tensión entre $0 \%$ y $20 \%$, recomendando un $10 \%$ de tensión en los casos de hematomas, estirando previamente la piel de la zona a tratar (19). El objetivo es que las tiras que se retraen hacia la base creando una elevación en la piel, asistan la eliminación del edema al redireccionar el fluido hacia una vía linfática menos congestionada por medio de los ganglios linfáticos; al mismo tiempo, al reducir la presión sobre el tejido muscular, se mejora la contracción, lo cual contribuye al peristaltismo del sistema ya que los músculos con sus contracciones ejercen un efecto de bomba sobre el sistema circulatorio sanguíneo y linfático (figura 9). 


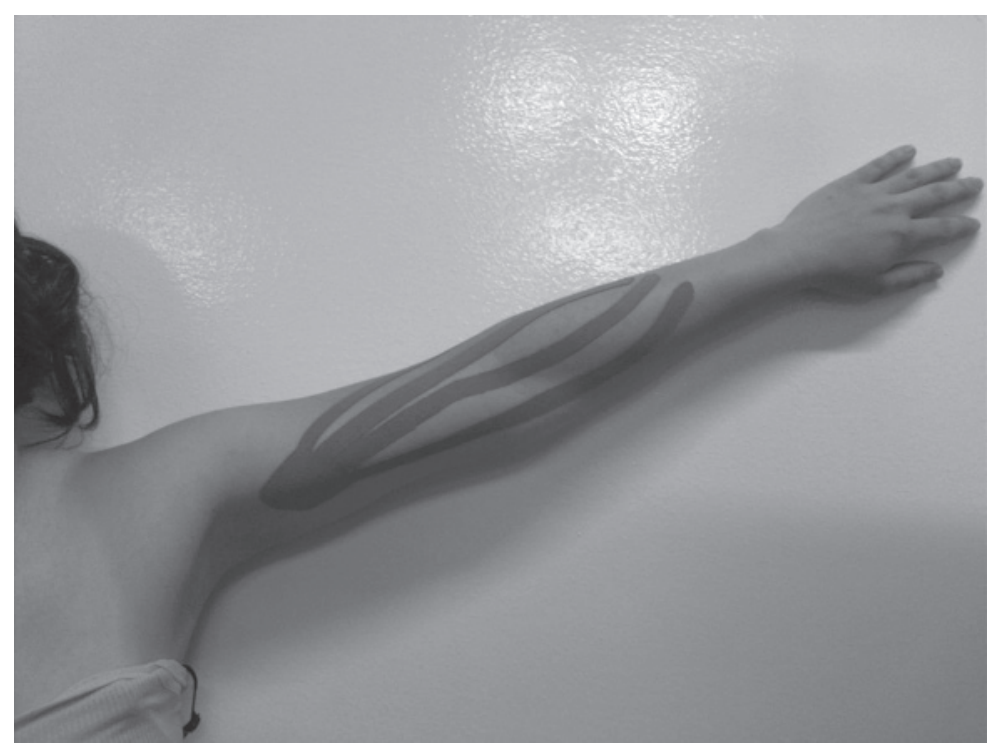

Figura 9. Técnica linfática

Fotografía: Universidad Manuela Beltrán.

El vendaje neuromuscular no pretende desplazar al masaje manual, la idea es que sean complementarios, pero sí hay que destacar que la acción del vendaje neuromuscular dura durante todo el tiempo que el paciente tiene aplicado el vendaje, con lo cual la estimulación circulatoria y de drenaje es permanente, lo que lo hace ideal como complemento a cualquier otra técnica de drenaje linfático.

\section{Conclusiones}

La piel puede ser considerada como el órgano sensorio más grande del cuerpo humano, aspecto que hace que a través de ella podamos influir mediante aferencias sensoriales al sistema nervioso central. La piel y las fascias comparten una misma inervación por lo que guardan una estrecha comunicación neuromecánica y responden a estímulos sensorios. El efecto neuromecánico del vendaje está dado por el reflejo de reposo neurogénico el cual se logra cuando el tejido celular subcutáneo y la fascia muscular, también se mueven en dirección a la base del vendaje, efecto que está mediado por la inervación conjunta de la epidermis, la lámina subcutánea, la fascia y el músculo.

Las fascias cumplen con muchas funciones en el organismo entre las que cabe resaltar para el caso la función de soporte, hemodinámica y de comunicación, siendo esta última la que permite que todos los tejidos conectivos como el tejido subcutáneo, los músculos, ligamentos, tendones y huesos estén interconectados por las fascias y trabajen en función sinergista. Este aspecto explica también cómo los estímulos aplicados por el vendaje a la piel se trasmiten también hacia los tejidos profundos.

El principal efecto que produce analgesia al aplicar el vendaje neuromuscular es que este forma elevaciones sobre la piel llamadas 'convoluciones' lo cual disminuye la presión intersticial y, por ende, la estimulación de los nociceptores. Los nociceptores son también influidos por el vendaje neuromuscular gracias a la normalización de la circulación sanguínea y a su evacuación linfática porque los síntomas dolorosos e inflamatorios dis- 
minuyen al drenar la acumulación de mediadores inflamatorios que sensibilizan los mecanoreceptores y nociceptores haciéndolos hiperexcitables.

Como el sistema vascular es interdependiente del sistema fascial y, además, las fascias posibilitan la circulación por sus contracciones, la acción de movilizar la epidermis sobre la dermis genera un espacio que disminuye inmediatamente la presión, el espacio conseguido por la elevación de la piel aumenta el intersticial, por lo cual no solo permite un mejor tránsito sanguíneo y linfático sino que le permite al tejido fascial recuperar su motilidad y función. Es muy importante tener en cuenta que para que haya una correcta aplicación del vendaje así como para que se puedan obtener los resultados esperados es indispensable que quienes lo apliquen conozcan la anatomía, fisiología y biomecánica del cuerpo humano.

Todas las anteriores conclusiones permiten un mayor acercamiento a la comprensión de la integración neuromotríz del sistema músculo -esquelético y tegumentario que está dado a través de las fascias, y en la manera como la aplicación del vendaje neuromuscular puede incidir sobre ellos.

\section{Agradecimientos}

La autora quiere expresar agradecimientos al Departamento de Investigaciones de la Universidad Manuela Beltrán seccional Bucaramanga, así como a su diseñador gráfico por el apoyo recibido durante la realización e ilustración del presente artículo.

\section{Descargos de responsabilidad}

La autora declara que la financiación de la presente investigación es totalmente institucional y corresponde al Departamento de Investigaciones de la Universidad Manuela Beltrán seccional Bucaramanga.

\section{Bibliografía}

1. Selva, F. El vendaje neuromuscular. Art. Divulgatio. 2008 marzo [citado 2013 jun 2]; IV(1):39. Disponible en: http://www.vendajeneuromuscular.es/publicaciones/UV-Fisioterapia-al-dia-vendajeneuromuscular.pdf

2. Aguirre T. Kinesiology Taping. Teoría y práctica. Andoain: Biocorp Europa; 2010.

3. Magaña M. Dermatología. México D. F.: Editorial Médica Panamericana; 2003.

4. Falabella R, Victoria J, Barona MI, Domínguez L. Dermatología. Bogotá: Colección para Investigaciones Biológicas; 2005

5. Paoletti S. El papel de los tejidos en la mecánica humana. 3. a ed. Barcelona: Editorial Paidotribo; 2004.

6. Smith-Agreda V, Ferres-Torres E. Principios de anatomo-fisio-patología. Barcelona: Editorial Paidotribo; 2004.

7. Pilat A. Terapias miofasciales: Inducción miofascial. 2003 [citado 2014 feb]. Disponible en: http://site. ebrary.com/lib/biblioumbsp/docDetail.action?docID=10498352\&p00=fascias

8. Mancuso P. El sistema Fascial. 2008 [citado: 2013 may]. Disponible en: http://www.odontotienda.com. ar/img/el_sistema_fascial.pdf

9. Martín E. Fundamentos de fisiología. Madrid: Editorial Paraninfo; 2006.

10. Fernández JM. Vendajes neuromusculares E.U. de fisioterapia. 2011 [citado 2013 abr. 5]. Disponible en: http://www.uclm.es/profesorado/jmfernandez/Alumnos/Tecnicas \%20Especiales/Vendaje \%20 Neuromuscular\%201.3\%20alumnos.pdf 
11. Guede F. Biomecánica de músculo. [citado 2013 abr. 5]. Disponible en: http://www.fcs.uner.edu.ar/ libros/archivos/articulos/biomecanica_de_musculo.pdf

12. Montaño P. Vendaje neuromuscular. Bases neurofisiológicas. [citado 2013 abr. 5]. Disponible en: http:// www.aevnm.com/docs/VNM-Bases_Neurofisiologicas \%5B1\%5D.pdf

13. Gordon MS. Fisiología animal comparada. Madrid: Editorial Continental; 1979.

14. Johansson H, Pederson J, Bergenheim M, Djupsjobacka M. Peripheral afferents of the knee: their effects on central mechanisms regulating muscle stiffness, joint stability, and proprioception and coordination. En: Lephart, Scott M, Fu FH, editor. Proprioception and neuromuscular control in joint stability. Champaign: Human Kinetics Publishers; 2000. p. 5-22.

15. Schutte MJ, Dabezies EJ, Zimny ML, Happel LT. Neural anatomy of the human anterior cruciate ligament. J Bone Joint Surg Am. 1987;69(2):243-7.

16. Kase K, Wallis J, Kase T. Clinical Therapeutic Applications of the Kinesio Taping Method. Tokyo: Ken Ikai Co Ltd; 2003.

17. Lephart SM, Riemann BL, Fu FH. Introduction to the sensoriomotor system. En: Lephart SM, Fu FH. Proprioception and neuromuscular control in joint stability. Champaign: Human Kinetics, 2000. p. 8-9

18. Fuhr AW. Activator Methods Chiropractic Technique. Missouri:Mosby Elsevier; 2007.

19. Sociedad Argentina de Terapia Intensiva. Terapia intensiva. 4. ${ }^{a}$ ed. Buenos Aires: Editorial Médica Panamericana; 2007.

20. Sijmonsma J. Manual de TNM. Cascais: Aneid Press; 2007.

21. Fernández JC. Fisiología Linfática. Errores de Interpretación. Patologías del Sistema Linfático. Fisiopatología Actual. En El Sistema Linfático. Historia, iconografía e implicaciones fisioterapéuticas. Madrid: Editorial Médica Panamericana; 2006.

22. Ciucci JL. Linfedema de miembro superior postratamiento de cáncer de mama. Buenos Aires: Editorial Nayarit; 2004 